\title{
Circular RNA profiling in plasma exosomes from patients with gastric cancer
}

\author{
MIN RAO ${ }^{1}$, YONGGANG ZHU ${ }^{2}$, LINGZHI QI ${ }^{3}$, FENG HU$^{1}$ and PUJUN GAO ${ }^{4}$ \\ ${ }^{1}$ Department of Hepatology and Gastroenterology, The Second Part of First Hospital of Jilin University, Changchun, \\ Jilin 130021; ${ }^{2}$ Department of Radiotherapy, China-Japan Union Hospital of Jilin University, Changchun, Jilin 130033; \\ ${ }^{3}$ Department of Gastroenterology, The People's Hospital of Jilin Province; ${ }^{4}$ Department of Hepatology, \\ The First Hospital of Jilin University, Changchun, Jilin 130021, P.R. China
}

Received February 14, 2020; Accepted May 27, 2020

DOI: $10.3892 / \mathrm{ol} .2020 .11800$

\begin{abstract}
Gastric cancer (GC) is among the most common cancer types worldwide with high mortality. Recent studies have shown that exosomes play a crucial role in the tumorigenesis of GC. The present study aimed to investigate the circular RNA (circRNA) profile in plasma exosomes from patients with gastric cancer (GC). Peripheral blood samples were collected from 5 patients with GC and 5 healthy donors, and exosomes were isolated from plasma. The high-throughput RNA sequencing (RNA-seq) method was applied to detect the differently expressed circRNAs (DE circRNAs). Subsequently, sequencing results were confirmed by reverse transcription quantitative (RT-q) PCR. The potential roles of DE circRNAs in GC were identified using Gene ontology (GO) and Kyoto Encyclopedia of Gene and Genome (KEGG) analysis. Furthermore, MiRanda software was used to predict circRNA-micro-RNA (miRNA) interactions. A total of 67,880 circRNAs were identified in all samples and 1,060 significantly DE circRNAs were screened, including 620 upregulated and 440 downregulated ones. These results were further confirmed by RT-qPCR. GO and KEGG analyses revealed that these circRNAs were significantly associated with 'cell cycle', 'cytoskeleton organization', 'cellular response to DNA damage', 'regulation of GTPase activity', 'phosphatidylinositol signaling pathway', 'MAPK signaling pathway', 'thyroid hormone signaling pathway', 'chemokine signaling pathway' and 'Wnt signaling pathway'. In addition, a circRNA-miRNA-mRNA interaction network was established. Taken together, these findings may help better understanding the underlying mechanisms of GC and identifying new
\end{abstract}

Correspondence to: Professor Pujun Gao, Department of Hepatology, The First Hospital of Jilin University, 71 Xinmin Street, Changchun, Jilin 130021, P.R. China

E-mail: pujungao@sina.com

Key words: gastric cancer, exosomes, circular RNA, high-throughput sequencing molecular alterations in GC, and allow the enrichment of the circRNA profiling in human GC.

\section{Introduction}

Gastric cancer (GC) is one of the most common types of cancer and is responsible for an estimated 783,000 deaths and over 1,000,000 new cases in 2018 worldwide, making it the fifth most frequently diagnosed cancer and the third leading cause of cancer-associated mortality (1). Surgical resection remains the most promising intervention towards localized GC tumors; however, many patients with advanced-stage GC present with inoperable disease at diagnosis or develop recurrent disease following resection (2). Patients with GC exhibit extremely poor quality of life and poor prognosis with a five-year survival rate $<15 \%$ (3). The development of sensitive and specific biomarkers is therefore crucial and may improve current management of GC, including early diagnosis, prognosis, recurrence monitoring and treatment response evaluation.

Recent studies have focused on the function of exosomes in local and systemic cell-to-cell communication in cancer progression and on their applications in cancer screening and diagnosis $(3,4)$. Exosomes are lipid bilayer vesicles of endocytic origin with a typical size of 30-100 nm, which are detected in most body fluids, including serum, urine and saliva (5). Exosomes are produced from various cell types, and tumor cells secrete excessive amounts of these vesicles compared with non-tumor normal cells (6). Furthermore, exosomes serve a pivotal role in mediating signaling transduction between neighboring or distant cells via delivering proteins, RNAs and DNAs (4). Comparing the contents of exosomes derived from GC patients with the content of exosomes from healthy individuals may provide a better understanding of the molecular mechanisms of GC tumorigenesis and progression and may help the identification of novel biomarkers for cancer development, metastasis and prognosis.

Circular RNAs (circRNAs) are a type of non-coding RNA (ncRNA) with a closed annular structure $(7,8)$. As an abundant cargo of exosomes, circRNAs can serve as biomarkers due to the following advantages: i) circRNAs are not easily degraded by $\mathrm{RNase} R$ due to their circular structure and may exist 
stably in exosomes; ii) circRNAs are highly conserved and tissue-, developmental stage- and tumor type-specific; and iii) some circRNAs are involved in tumorigenesis and associated with treatment response or patients' survival (9). A study of circRNAs expression in GC and paracancerous tissues identified 467 significantly differentially expressed circRNAs (DE circRNAs), including 214 upregulated and 253 downregulated ones (10). However, the expression profile of circRNAs in circulating exosomes from patients with GC remains unknown. In the present study, the RNA sequencing (RNA-seq) technique was used to detect exosomal circRNAs in the plasma from patients with GC and healthy donors and screen DE circRNAs. The potential roles of these DE circRNAs and their target miRNAs were predicted using bioinformatics methods.

\section{Materials and methods}

Patients and sample collection. In the present study, 5 patients with GC were recruited from the First Hospital of Jilin University between December 2018 and February 2019. All patients were pathologically diagnosed with gastric adenocarcinoma and were free of any other types of cancer. Patients who had received any type of anticancer treatment were excluded from the present study. In addition, 5 healthy donors with no history of cancer were enrolled as healthy controls (HC). The clinicopathological characteristics of patients and volunteers are presented in Table I. In total, $5 \mathrm{ml}$ of peripheral blood samples were collected intravenously from each individual in BD Vacutainer ${ }^{\circledR}$ Venous Blood Collection Tubes containing EDTA, and plasma was isolated and frozen immediately at $-80^{\circ} \mathrm{C}$ until use. This study was approved by the local independent Ethics Committee and the Institutional Review Board of the First Hospital of Jilin University (Changchun, China), and informed consent was obtained from all patients and volunteers. Blood collection and experiments were performed in accordance with the Declaration of Helsinki and relevant guidelines and regulations.

Exosome isolation and identification. Exosomes were isolated from $1 \mathrm{ml}$ of plasma samples pre-filtered by passing through a $0.8 \mu \mathrm{m}$ filter using an exoEasy Maxi kit (Qiagen, Inc.) according to the manufacturers' instructions and frozen immediately at $-80^{\circ} \mathrm{C}$ until use.

Exosomes were visualized by transmission electron microscope (TEM) as previously described (11). Briefly, following loading onto carbon-coated formvar grids, and staining with $2 \%$ uranyl acetate for $10 \mathrm{~min}$ at room temperature and lead citrate for $5 \mathrm{~min}$ at room temperature, exosomes were visualized using TEM (FEI TF20, Thermo Fisher Scientific, Inc.) with a magnification of $x 75,000$.

Western blotting was used to detect the typical exosomal protein markers, CD9 and TSG101 (12). Exosomes were lysed on ice in radioimmunoprecipitation assay buffer (Beyotime Institute of Biotechnology,) supplemented with $1 \%$ protease inhibitor cocktail (Cell Signaling Technology). Following protein quantification using a BCA Protein Assay kit (Pierce; Thermo Fisher Scientific, Inc.), $30 \mu \mathrm{g}$ exosomal proteins were separated by $12 \%$ SDS-PAGE and transferred onto a polyvinylidene fluoride membrane. Membranes were blocked with TBS-5\% BSA at room temperature for $2 \mathrm{~h}$ and incubated with the primary antibodies rabbit anti-CD9 (cat. no. D8O1A; 1:1,000; Cell Signaling Technology, Inc.), rabbit anti-tumor susceptibility gene 101 (TSG101; cat. no. ab125011; 1:1,000; Abcam) and rabbit anti-calnexin (cat. no. C5C9; 1:1,000, Cell Signaling Technology, Inc.) at $4^{\circ} \mathrm{C}$ overnight. HRP-Goat Anti-Rabbit IgG (cat. no. 111-035-003; Jackson ImmunoResearch Laboratories, Inc.) was used as the secondary antibody at 1:2,000 dilution and incubated for $2 \mathrm{~h}$ at room temperature. A EasyBlot ECL kit (cat. no. D601039-0050; Sangon Biotech Co., Ltd.) was used to visualize the bands. CD9 and TSG101 are two of the most frequently identified markers in exosomes, whereas calnexin is an endoplasmic reticulum protein that is used as a negative control.

RNA isolation and quality control. Total RNA was extracted from exosomes using TRIzol reagent (Life Technologies; Thermo Fisher Scientific, Inc.). The quality control and quantification of the RNA samples were performed using the NanoDrop ND 1000 spectrophotometer (Thermo Fisher Scientific, Inc.). RNA integrity and DNA contamination were assessed by electrophoresis on a $1 \%$ denaturing agarose gel. Only DNA-free RNA samples with $\mathrm{OD}_{260} / \mathrm{OD}_{280}$ ratio between 1.8 and 2.1 and RNA integrity number (RIN) $\geq 7$ were used for further experiments.

RNA library construction and circRNA sequencing. High-throughput RNA-seq was performed by Cloud-Seq Biotech, Inc. Briefly, the NEBNext ${ }^{\circledR}$ rRNA Depletion Kit (New England BioLabs, Inc.) was used to remove ribosomal RNAs (rRNAs) from total exosomal RNAs according to the manufacturers' instructions. Subsequently, RNA libraries were constructed from rRNA-depleted RNAs using the NEB Next ${ }^{\circledR}$ Ultra $^{\mathrm{TM}}$ II Directional RNA Library Prep Kit (New England BioLabs, Inc.) according to the manufacturers' protocol. The quality and quantity of the RNA libraries were evaluated using the BioAnalyzer 2100 system (Agilent Technologies, Inc.). These libraries were then sequenced on an Illumina HiSeq 4000 sequencer (Illumina, Inc.) and $150 \mathrm{bp}$ paired-end reads were generated. Quality score of $30(\mathrm{Q} 30)$ was used to determine sequencing quality. Furthermore, cutadapt software (v1.9.2) (13) was used to trim 3' adaptor sequences and to remove low-quality reads to acquire clean reads, which were then aligned to the reference genome/transcriptome via the Bowtie2 software (v2.2.4) (14). In addition, circRNAs were identified with the find_circ software (v1.2) (15). Finally, raw junction reads for each sample were normalized to the number of total reads and the expression levels of circRNAs were transformed into $\log$ CPM values ( $\log 2$ counts per million; normalized for library sizes).

Identification of DE circRNAs. Differential expression of circRNAs was calculated using the edgeR package of the $\mathrm{R}$ software (v3.3.2). DE circRNAs were identified through fold-change ( $\mathrm{FC} \geq 2.0$; i.e. $\log _{2} \mathrm{FCl}>1$ ) between $\mathrm{GC}$ cases and controls, and t-tests $(\mathrm{P}<0.05)$. DE circRNAs with $\log _{2} \mathrm{FC}<0$ and $\log _{2} \mathrm{FC}>0$ were considered as down- and upregulated genes, respectively. Volcano plot visualization based on false discovery rate (FDR)-adjusted P-values was performed using ggplot2 in R software (v3.5.1). Subsequently, a hierarchical heatmap was constructed to reveal circRNAs expression 
Table I. Clinicopathological characteristics of patients with GC and healthy volunteers.

\begin{tabular}{|c|c|c|c|c|c|}
\hline Sample ID & Age, years & Sex & Histological type & Histological grade & TNM stage \\
\hline \multicolumn{6}{|l|}{ GC } \\
\hline GC190121 & 71 & Male & Adenocarcinoma & Poorly & T4aN3aM0 IIIB \\
\hline GC190122 & 58 & Male & Adenocarcinoma & Poorly & T2N1M0 IIA \\
\hline GC190124 & 63 & Male & Adenocarcinoma & Poorly & T3N2MO IIIA \\
\hline GC190125-2 & 64 & Male & Adenocarcinoma & Poorly & T4aN3aM0 IIIB \\
\hline GC190128-2 & 65 & Male & Adenocarcinoma & Poorly & T3N3aMO IIIB \\
\hline \multicolumn{6}{|l|}{$\mathrm{HC}$} \\
\hline HC190128-1 & 33 & Male & & & \\
\hline HC190128-2 & 43 & Male & & & \\
\hline HC190128-3 & 29 & Male & & & \\
\hline HC190128-4 & 26 & Male & & & \\
\hline HC190128-5 & 37 & Male & & & \\
\hline
\end{tabular}

HC, healthy control; GC, gastric cancer; TNM, Tumor-Node-Metastasis.

pattern among samples via the heatmap. 2 function of the gplots package in R software (v3.5.1).

Gene ontology (GO) and Kyoto Encyclopedia of Gene and Genome (KEGG) pathway enrichment analyses. DE circRNAs were submitted to The Database for Annotation, Visualization and Integrated Discovery (DAVID: http://david. abcc.ncifcrf.gov) to unveil their potential roles (16) via GO (17) and KEGG (18) enrichment analysis. The P-value $\left(-\log _{10}\right)$ scores indicated the significance of GO terms and KEGG pathway correlations. The top 10 enriched GO terms, including molecular function (MF), cellular component (CC) and biological process (BP), and the top $10 \mathrm{KEGG}$ pathways of upregulated and downregulated circRNAs were presented via enrichment scores. Finally, the pathway relation network was established using the Cytoscape software (v3.6.1) based on the significantly enriched KEGG pathways.

Reverse transcription quantitative PCR (RT-qPCR). RT-qPCR was performed to verify the expression levels of the selected DE circRNAs in plasma exosomes derived from patients with GC and healthy controls. Total RNA was reverse transcribed to cDNA using SuperScriptTM III Reverse Transcriptase (Invitrogen; Thermo Fisher Scientific, Inc.) according to the manufacturers' protocol. Subsequently, the cDNA library of each sample was subjected to RT-qPCR reactions on a QuantStudio 5 Real-Time PCR System (Thermo Fisher Scientific, Inc.) using a qPCR SYBR Green master mix (CloudSeq Biotech, Inc.). Primer sequences of the randomly selected circRNAs and GAPDH (internal control) are listed in Table II (Invitrogen; Thermo Fisher Scientific, Inc.). The circRNA expression levels were normalized using GAPDH as the reference gene and calculated by applying the $2^{-\Delta \Delta C q}$ method (19). Each circRNA sample was analyzed in triplicate.

Prediction of circRNA-miRNA-mRNA interaction. CircRNA-miRNA interaction was predicted using the MiRanda software (August 2010 release) (20). Subsequently, top 5 putative target miRNAs and their binding sites on DE circRNAs were identified. In addition, the downstream target mRNAs of miRNAs were predicted using TargetScan (http://www.targetscan.org) (21). Finally, the potential functional DE circRNA-miRNA-mRNA network was visualized with Cytoscape software (v3.6.1) (22).

Statistical analysis. Statistical analysis was performed using SPSS 22.0 software (IBM Corp.). Student's t-test was used to evaluate the differences in circRNAs expression levels between patients with GC and healthy controls. RT-qPCR data were expressed as the means \pm standard deviation of the mean. $\mathrm{P}<0.05$ was considered to indicate a statistically significant difference.

\section{Results}

Identification of isolated exosomes. Exosomes were isolated from the plasma of patients with GC and healthy donors. The morphology and size of the obtained exosomes were identified using TEM. Exosomes ranged from 30 to $100 \mathrm{~nm}$ in size and exhibited a cup-shaped morphology with a clearly defined and relatively intact membrane (Fig. 1A). The exosomal proteins CD9 and the endosomal sorting complexes required for transport TSG101 were detected in all samples by western blotting. These results confirmed the exosomal characteristics of these extravesicles. In addition, the endoplasmic reticulum marker calnexin was undetectable in these exosomes, confirming the purity of the isolation (Fig. 1B).

$D E$ circRNAs in plasma exosomes from patients with $G C$. The profiling of circRNAs expression in the circulating exosomes derived from patients with GC and healthy controls was performed using high-throughput RNA-seq. A total of $732,993,440$ raw and 699,178,882 clean reads were obtained, and 67,880 circRNAs were identified in all samples. The number of detected reads and circRNAs in each sample is listed in Table III. The predicted length of circRNAs ranged 
Table II. Randomly selected circRNAs for RT-qPCR and primer sequences.

\begin{tabular}{|c|c|c|c|}
\hline No. & CircRNA ID & Primer name & Primer sequence \\
\hline 1 & chr2:61468695-61484485- & $\begin{array}{l}\text { 2-Forward } \\
\text { 2-Reverse }\end{array}$ & $\begin{array}{l}\text { GCATGGAACCAGAGGAAGAA } \\
\text { CAGAGGCTGCTTATCCATGG }\end{array}$ \\
\hline 2 & chr4:39915231-39927553- & $\begin{array}{l}\text { 10-Forward } \\
\text { 10-Reverse }\end{array}$ & $\begin{array}{l}\text { GCAATGATGGAGAAGAGCGA } \\
\text { TGCAATCTTCCAATTCAAAGCAG }\end{array}$ \\
\hline 3 & chr17:35797839-35800763+ & $\begin{array}{l}\text { 7-Forward } \\
\text { 7-Reverse }\end{array}$ & $\begin{array}{l}\text { TGCTGAACCTGAAACAAGCA } \\
\text { CCAAAGCCACAGTCCATCAC }\end{array}$ \\
\hline Internal standard & GAPDH & $\begin{array}{l}\text { IS-Forward } \\
\text { IS-Reverse }\end{array}$ & $\begin{array}{l}\text { GGCCTCCAAGGAGTAAGACC } \\
\text { AGGGGAGATTCAGTGTGGTG }\end{array}$ \\
\hline
\end{tabular}

Table III. Number of detected reads and circRNAs in each sample.

\begin{tabular}{lccccc}
\hline Sample & Raw reads & Q30 $(\%)$ & Clean reads & Clean ratio $(\%)$ & CircRNA number \\
\hline GC190121 & $79,522,714$ & 85.51 & $78,394,480$ & 98.58 & 8,585 \\
GC190122 & $70,645,332$ & 83.05 & $69,090,516$ & 97.80 & 6,210 \\
GC190124 & $81,482,080$ & 80.57 & $71,190,454$ & 87.37 & 7,893 \\
GC190125-2 & $74,364,070$ & 82.04 & $72,423,264$ & 97.39 & 8,994 \\
GC190128-2 & $74,726,070$ & 82.57 & $70,623,532$ & 94.51 & 1,690 \\
HC190128-1 & $76,588,480$ & 82.48 & $73,503,134$ & 95.97 & $50,075,766$ \\
HC190128-2 & $72,454,750$ & 81.14 & $69,163,734$ & 95.46 & $46,694,522$ \\
HC190128-3 & $75,169,572$ & 83.23 & $73,402,884$ & 97.65 & $51,661,182$ \\
HC190128-4 & $70,163,844$ & 86.56 & $61,296,786$ & 87.36 & $38,213,918$ \\
HC190128-5 & $70,567,646$ & 82.58 & $68,197,198$ & 96.64 & $47,517,420$ \\
\hline
\end{tabular}

${ }^{\mathrm{a}} \mathrm{Q} 30$ refers to the percentage of nucleotides with Phred quality score $>30$. GC, gastric cancer; HC, healthy control.
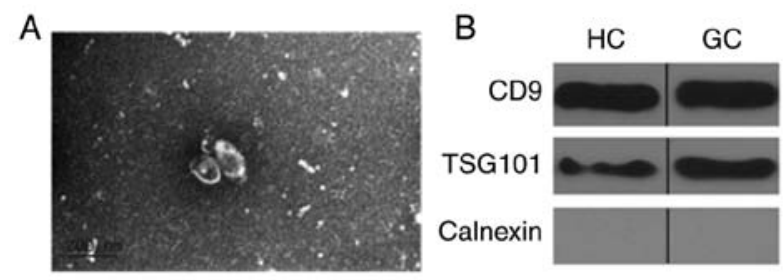

Figure 1. Identification of exosomes. (A) Representative TEM images of exosomes isolated from. Scale bar, $200 \mathrm{~nm}$. (B) Western blotting analysis of exosomes. CD9 and TSG101 were detected in exosome samples and calnexin was used as negative control. TEM, transmission electron microscopy; CD9, cluster of differentiation 9; TSG101, tumor susceptibility gene 101.

between 53 and 99,588 nt with an average size of 4,176 nt. The length distribution of the circRNAs is presented in Fig. 2A. According to their association with protein-coding genes, circRNAs were classified into the following five categories: 22,656 circRNAs were exonic; 3,728 circRNAs were intronic; 306 circRNAs were intergenic; 1,406 circRNAs were antisense; and 4,997 were sense overlapping (Fig. 2B). The raw read counts were normalized and DE circRNAs were filtered using edgeR. Subsrequently, 1,060 significantly DE circRNAs with $\mathrm{FC} \geq 2.0$ and $\mathrm{P}<0.05$ were identified (Fig. $2 \mathrm{C}$ ), including 620 upregulated (Table SI) and 440 downregulated ones
(Table SII). The top 5 up- and downregulated circRNAs in patients with are listed in Table IV. Eventually, the heatmap of circRNA expression profiles in all samples revealed a remarkably distinguishable exosomal circRNAs expression pattern in GC cases compared with HC (Fig. 2D).

Validation of DE circRNAs expression by RT-qPCR. RT-qPCR was performed to verify the differential expression of circRNAs. Among the high-throughput RNA-seq results, two upregulated (chr2: 61468695-61484485- and chr4: 39915231-39927553-) and one unaffected circRNA (chr17: 35797839-35800763+) were randomly selected for validation. The circRNA expression levels were normalized using GAPDH as the internal control. The results demonstrated that the expression levels of chr2: 61468695-61484485- and chr4: 39915231-39927553- were significantly upregulated in samples from patients with GC compared with HC. In addition, the expression of chr17: 35797839-35800763+ was comparable between the two groups (Fig. 3), suggesting that the observed circRNA expression profiles were reliable.

GO and KEGG enrichment analyses of DE circRNAs. To reveal the biological properties and functions of DE circRNAs, GO and KEGG analyses were carried out. Furthermore, DAVID website was used to visualize the DE circRNAs enrichment 

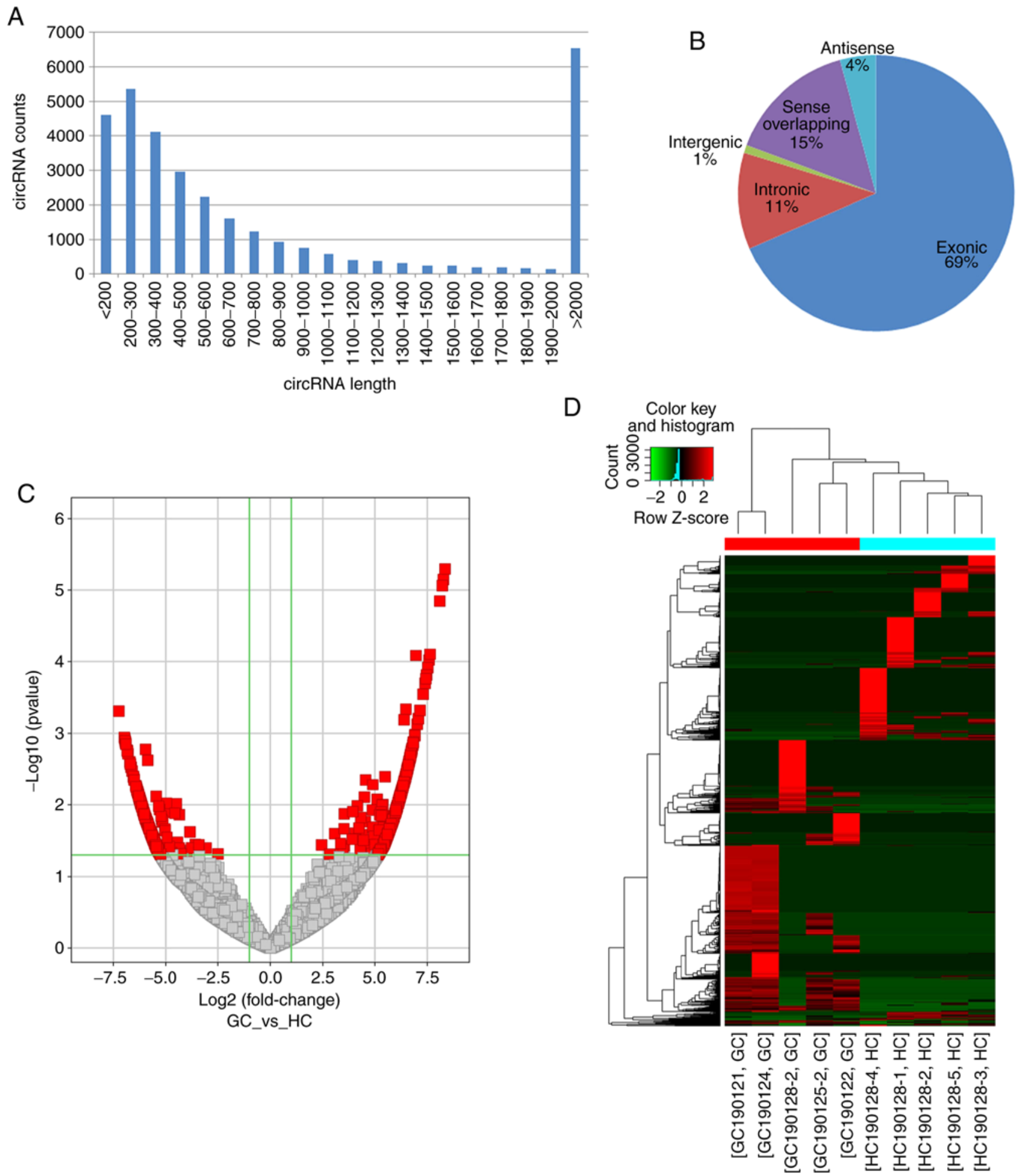

Figure 2. Expression patterns circRNAs in plasma exosome from patients with GC and HC identified by high throughput RNA sequencing. (A) Length distribution of detected circRNAs. (B) Categories of circRNAs. (C) Volcano plot showing DE circRNAs between GC cases and HCs. Red dots [llog2FCl $>1,-\log 10$ (P-values) $>1.3$ ] represent significantly upregulated $(\log 2 \mathrm{FC}>1)$ and downregulated $(\log 2 \mathrm{FC}<-1)$ circRNAs. (D) Hierarchical cluster analysis and heatmap of DE circRNAs with a P-value $<0.05(n=5)$. Each row represents the expression level of a circRNA and each column represents an exosome sample. Pseudo-colors indicate expression levels from low to high (green to red). circRNA, circular RNA; FC, fold change; GC, gastric cancer; HC, healthy control; DE, differentially expressed.

for the associated GO terms and KEGG pathways. Regarding the MF, upregulated DE circRNAs were associated with 'purine nucleotide binding', especially with 'adenyl nucleotide binding', 'ATP binding', 'protein binding', 'drug binding', 'transferase activity, transferring phosphorus-containing groups' and 'protein kinase activity', while the downregulated ones were associated with 'enzyme binding', especially 'GTPase binding', 'protein binding', 'ATP binding' and 'GTPase regulator activity'. For GO CC, upregulated DE circRNAs were significantly enriched in 'intracellular part', 

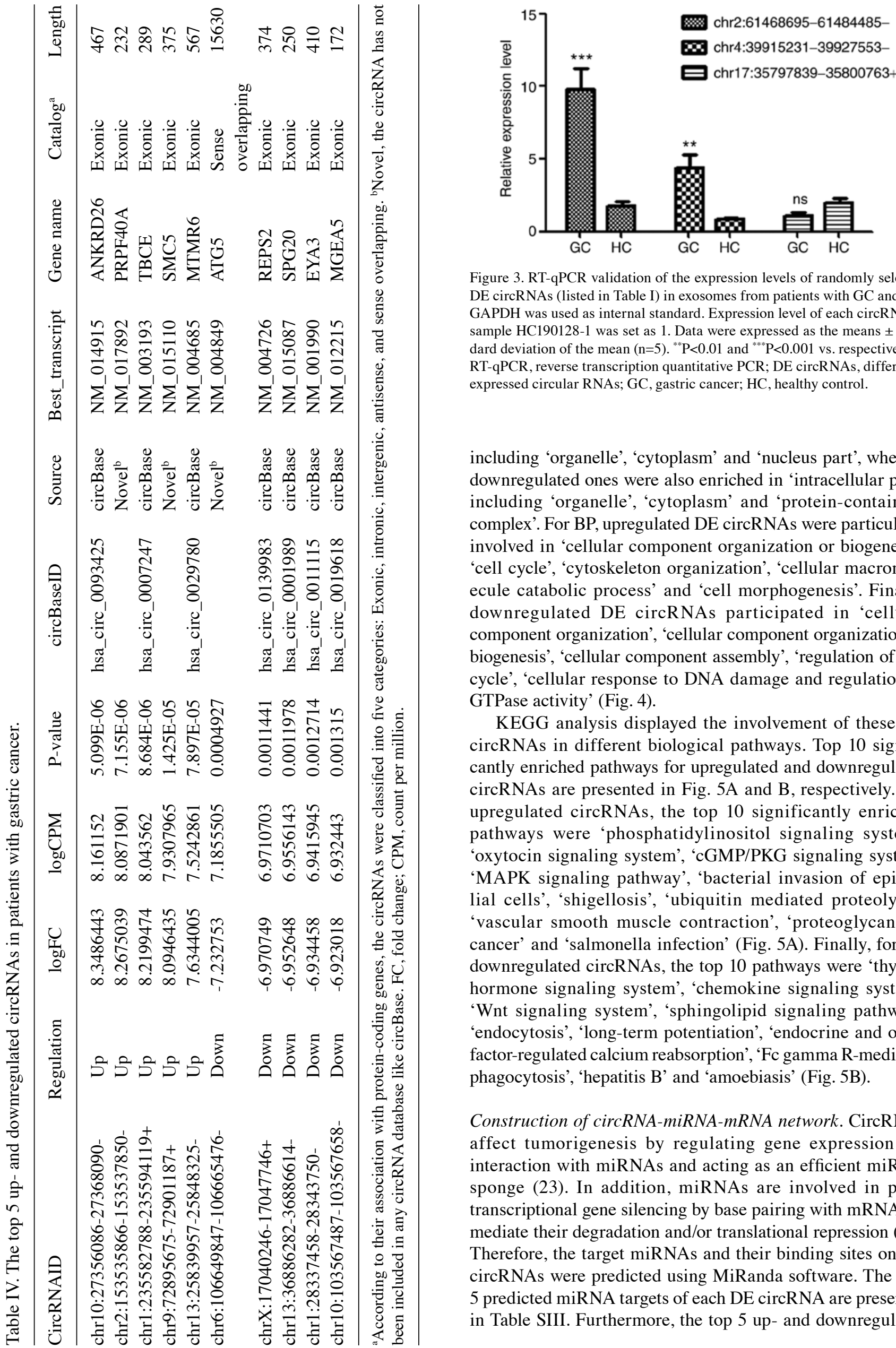

Figure 3. RT-qPCR validation of the expression levels of randomly selected DE circRNAs (listed in Table I) in exosomes from patients with GC and HC. GAPDH was used as internal standard. Expression level of each circRNA in sample HC190128-1 was set as 1. Data were expressed as the means \pm standard deviation of the mean $(n=5) .{ }^{* *} \mathrm{P}<0.01$ and ${ }^{* * * *} \mathrm{P}<0.001$ vs. respective HC. RT-qPCR, reverse transcription quantitative PCR; DE circRNAs, differently expressed circular RNAs; GC, gastric cancer; HC, healthy control.

including 'organelle', 'cytoplasm' and 'nucleus part', whereas downregulated ones were also enriched in 'intracellular part', including 'organelle', 'cytoplasm' and 'protein-containing complex'. For BP, upregulated DE circRNAs were particularly involved in 'cellular component organization or biogenesis', 'cell cycle', 'cytoskeleton organization', 'cellular macromolecule catabolic process' and 'cell morphogenesis'. Finally, downregulated DE circRNAs participated in 'cellular component organization', 'cellular component organization or biogenesis', 'cellular component assembly', 'regulation of cell cycle', 'cellular response to DNA damage and regulation of GTPase activity' (Fig. 4).

KEGG analysis displayed the involvement of these DE circRNAs in different biological pathways. Top 10 significantly enriched pathways for upregulated and downregulated circRNAs are presented in Fig. 5A and B, respectively. For upregulated circRNAs, the top 10 significantly enriched pathways were 'phosphatidylinositol signaling system', 'oxytocin signaling system', 'cGMP/PKG signaling system' 'MAPK signaling pathway', 'bacterial invasion of epithelial cells', 'shigellosis', 'ubiquitin mediated proteolysis', 'vascular smooth muscle contraction', 'proteoglycans in cancer' and 'salmonella infection' (Fig. 5A). Finally, for the downregulated circRNAs, the top 10 pathways were 'thyroid hormone signaling system', 'chemokine signaling system', 'Wnt signaling system', 'sphingolipid signaling pathway', 'endocytosis', 'long-term potentiation', 'endocrine and other factor-regulated calcium reabsorption', 'Fc gamma R-mediated phagocytosis', 'hepatitis B' and 'amoebiasis' (Fig. 5B).

Construction of circRNA-miRNA-mRNA network. CircRNAs affect tumorigenesis by regulating gene expression via interaction with miRNAs and acting as an efficient miRNA sponge (23). In addition, miRNAs are involved in posttranscriptional gene silencing by base pairing with mRNAs to mediate their degradation and/or translational repression (24). Therefore, the target miRNAs and their binding sites on DE circRNAs were predicted using MiRanda software. The first 5 predicted miRNA targets of each DE circRNA are presented in Table SIII. Furthermore, the top 5 up- and downregulated 
A
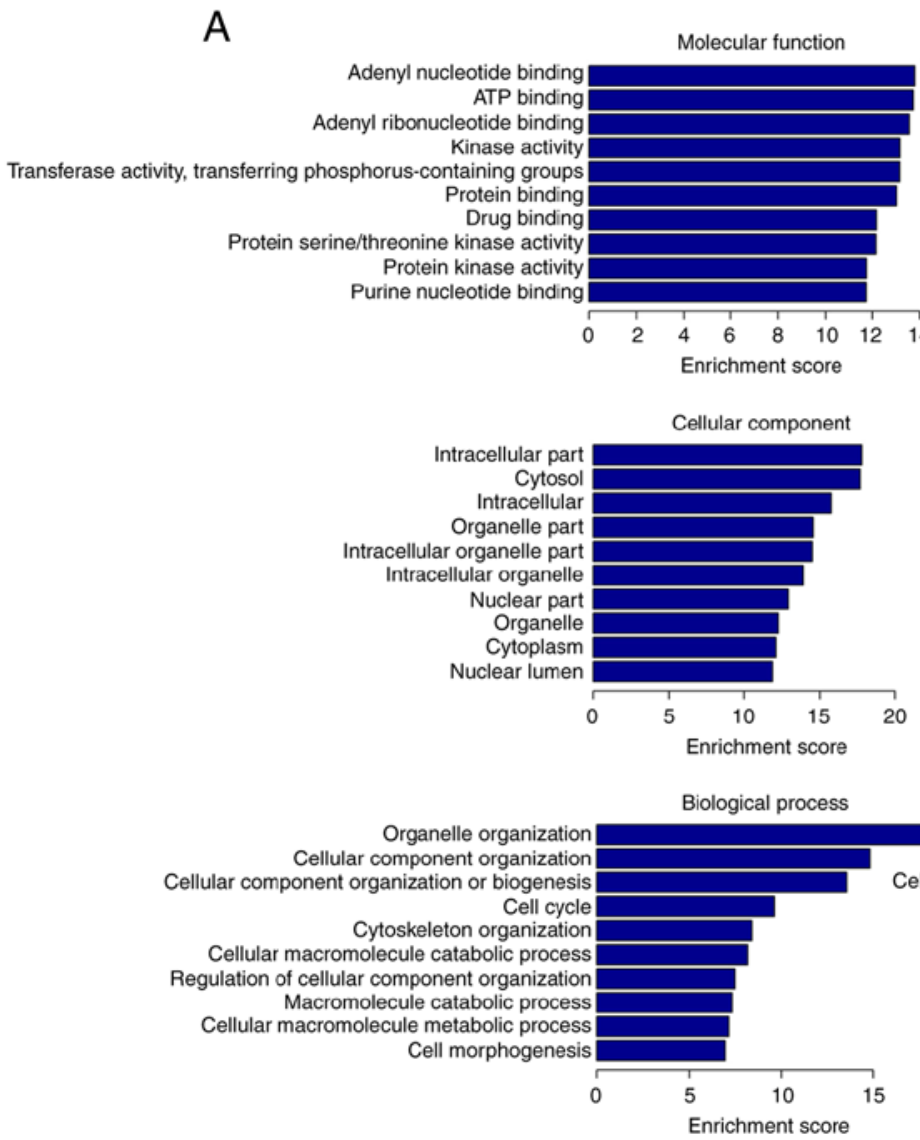

B
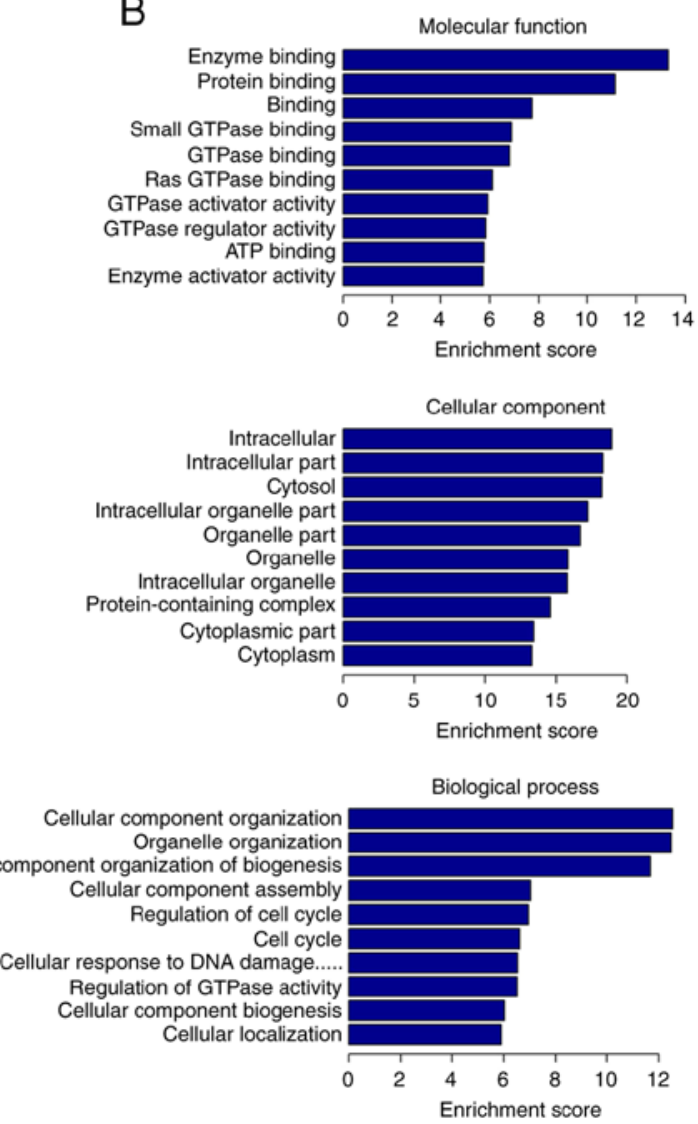

Figure 4. GO terms of DE circRNAs with top 10 enrichment score. Enrichment score was calculated as - $\log _{10}$ (P-value). (A) GO analysis of upregulated circRNAs. (B) GO analysis of downregulated circRNAs. GO, Gene ontology; DE circRNAs, differently expressed circular RNAs.

A

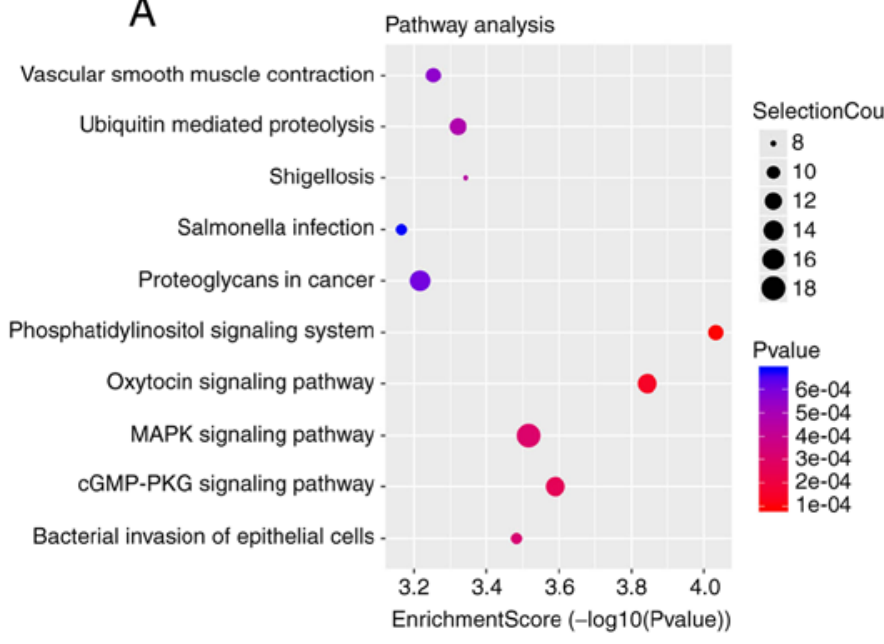

B

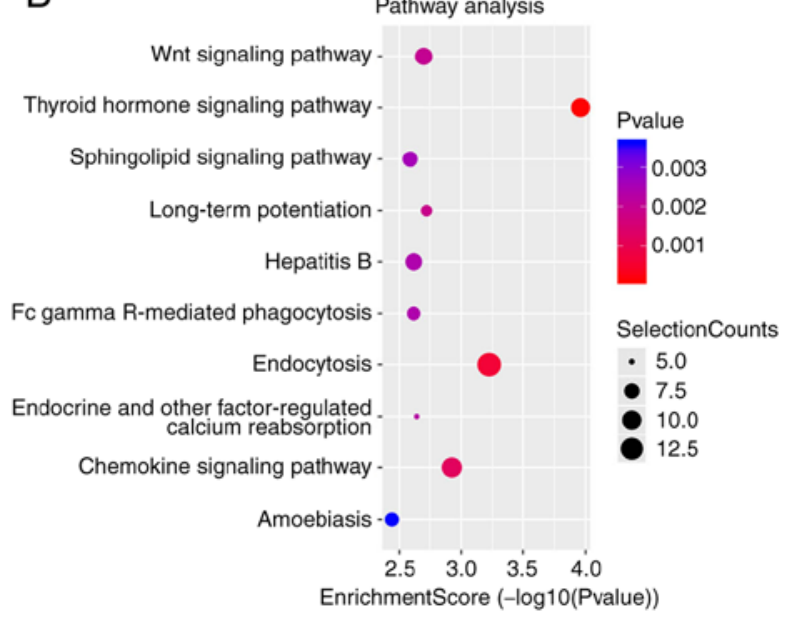

Figure 5. KEGG pathways of DE circRNAs with top 10 enrichment score. Enrichment score was calculated as - $\log _{10}$ (P-value). Selection counts represent the number of entities of the DE circRNAs directly associated with the listed pathways. (A) KEGG pathway analysis of upregulated circRNAs. (B) KEGG pathway analysis of downregulated circRNAs. KEGG, Kyoto Encyclopedia of Gene and Genome; DE circRNAs, differently expressed circular RNAs.

circRNAs as listed in Table IV and were used to construct the circRNA-miRNA network (Fig. 6).

\section{Discussion}

GC remains one of the most common and lethal types of cancer worldwide. Despite the declining incidence rate and advances in diagnosis, GC causes $>700,000$ deaths annually (1). Clarifying the underlying mechanisms of GC pathogenesis and developing sensitive and specific biomarkers are therefore required to improve GC management.

Exosomes represent an important group of extracellular vesicles, which have crucial roles in the initiation, progression and metastasis of several types of cancer, including GC (3). 


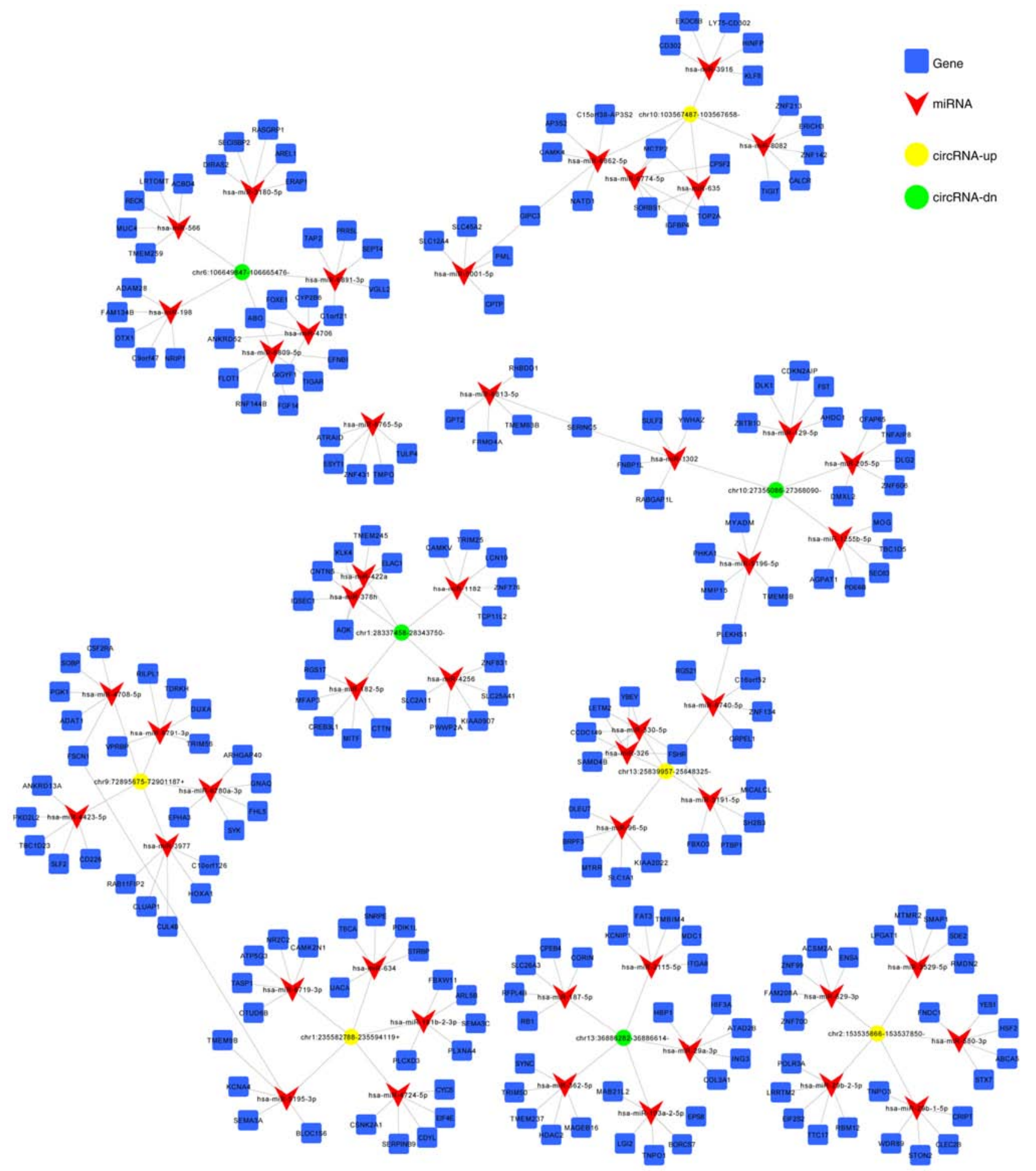

Figure 6. CircRNA-miRNA-mRNA network containing high-score interactions. CircRNA, miRNA and mRNA are represented by yellow circles, green triangles and blue squares, respectively. CircRNA, circular RNA; miRNA, micro-RNA; mRNA, messenger RNA.

In addition, exosomes are commonly found in various body fluids and may also exhibit a potential role in early diagnosis and evaluation of therapeutic effects of drugs in patients with GC (25). As a key class of exosomal cargos, circRNAs mediate tumor and stromal communication and are considered as emerging biomarkers for cancer diagnosis (5). In the present study, the expression profiles of exosomal circRNAs were compared between GC patients and $\mathrm{HC}$ using high-throughput RNA-seq. The findings from the present study may contribute to the identification of novel biomarkers for GC and may provide insights for future studies to investigate the mechanisms of GC pathogenesis.

A total of 1,060 DE circRNAs between GC patients and $\mathrm{HC}$ were identified, including 620 upregulated and 440 downregulated ones. The selected circRNAs were further evaluated using RT-qPCR and the results confirmed the reliability of the RNA-seq analysis. Furthermore, GO and KEGG enrichment analyses were performed to gain insights 
into the potential functions of theses DE circRNAs in GC oncogenesis.

The results from GO analysis revealed that DE circRNAs were mainly located in the intracellular cytoplasm and organelle and were associated with several important biological processes involved in tumorigenesis, including cell cycle, cytoskeleton organization, cellular response to DNA damage and regulation of GTPase activity. The members of small GTPase family, including RhoA and RhoC, have been associated with the progression of $\mathrm{GC}$ via regulation of cell proliferation, invasion and metastasis of GC cells. They regulate the proliferation, invasion and metastasis of $\mathrm{GC}$ and has potential clinical significance (26-28).

The majority of signaling pathways enriched in KEGG analysis, including phosphatidylinositol (29-31), MAPK (32), thyroid hormone $(33,34)$, chemokine $(6,35,36)$ and Wnt $(37,38)$ signaling pathways, are also associated with GC progression and chemotherapy resistance.

The phosphatase and tensin homolog (PTEN)/phosphatidylinositol 3-kinase (PI3K)/protein kinase B (Akt) signaling pathway serves a critical role in several cellular processes, including cell proliferation, apoptosis and invasion (29). Furthermore, PTEN/PI3K/Akt is the second most commonly altered pathway in human cancer following p53 pathway, with a frequency of 30-60\% among different tumor types (30). It has been reported that GC harbors one of the highest rates of oncogenic alterations in PI3K, which confers a therapeutic potential to the PI3K pathway (31).

MAPK signaling pathway has been implicated in the invasion and metastasis of GC cells (32), whereas Wnt signaling pathway serves a key role in cell proliferation during both normal and cancerous gut development $(37,38)$. It has also been reported that both pathways, as well as PI3K, are activated following Helicobacter pylori infection, resulting in increased expression of inflammatory cytokines and changes in apoptosis, proliferation and differentiation. These changes ultimately lead to the transformation of normal epithelial cells into oncogenic ones (39).

Thyroid hormone signaling has been characterized as a major effector of growth and homeostasis of the digestive system. Based on this finding, several studies have demonstrated that the occurrence of GC is associated with alterations in the protein levels of thyroid hormone receptor TR $\alpha$, autoimmune thyroid disease and goiter, suggesting a potential role of thyroid hormone signaling pathway in $\operatorname{GC}(33,34)$.

Several chemokines secreted by cancer cells and tumor-associated stromal cells are involved in metastatic tumor microenvironment (6). In addition, the chemokine signaling pathway has been shown to be associated with the survival, proliferation, angiogenesis and metastasis of tumor cells. Therefore, therapeutic strategies blocking the chemokine signaling pathway, including $\mathrm{C}-\mathrm{C}$ chemokine ligand $5 / \mathrm{C}-\mathrm{C}$ chemokine receptor type 5 and $\mathrm{C}-\mathrm{X}-\mathrm{C}$ motif chemokine ligand 12/C-X-C chemokine receptor type 4 axes, have been considered as effective strategies in the treatment of $\mathrm{GC}(35,36)$.

CircRNAs may control GC progression by regulating the expression of the key components of the aforementioned signaling pathways. As suggested by a previous study that screened GC tissues and adjacent tissues for differences in mRNAs and circRNAs expression using Agilent microarray technology, DE circRNAs had corresponding miRNA binding sites, and these circRNAs regulated the expression of target genes through interactions with miRNAs (10). Therefore the present study identified the putative miRNA targets of DE circRNAs and constructed the circRNA-miRNA-mRNA network to improve the general understanding on the role of circRNAs in regulating the expression of specific genes.

In summary, the present study identified a series of DE circRNAs in exosomes isolated from the plasma of patients with GC and $\mathrm{HC}$ by using high-throughput RNA-seq analysis. Furthermore, the potential functions of DE circRNAs were predicted using bioinformatics analysis. The results of the present study may contribute to uncover the underlying mechanisms of GC oncogenesis and help the development of targeted therapies and predictive biomarkers for GC diagnosis.

\section{Acknowledgements}

Not applicable.

\section{Funding}

This research was supported by the Research Fund of the First Hospital of Jilin University (grant no. 20170142) and the Natural Science Foundation of Science and Technology Department of Jilin Province (20200201496JC).

\section{Availability of data and materials}

The datasets used and/or analyzed during the current study are available from the corresponding author on reasonable request.

\section{Authors' contributions}

PG and MR conceived and designed the experiments, and wrote the article. MR, YZ and LQ performed the experiments. MR, FH and PG analyzed the data. All authors read and approved the final manuscript.

\section{Ethics approval and consent to participate}

All participants in this study gave informed consent according to the Helsinki Declaration, and all experimental protocols were approved by the Ethics Committee of the First Hospital of Jilin University (Changchun, China) (approval no. 2019-070).

\section{Patient consent for publication}

Not applicable.

\section{Competing interests}

The authors declare that they have no competing interests.

\section{References}

1. Bray F, Ferlay J, Soerjomataram I, Siegel RL, Torre LA and Jemal A: Global cancer statistics 2018: GLOBOCAN estimates of incidence and mortality worldwide for 36 cancers in 185 countries. CA Cancer J Clin 68: 394-424, 2018. 
2. Zhang T, Liu C, Huang S, Ma Y, Fang J and Chen Y: A down modulated MicroRNA profiling in patients with gastric cancer. Gastroenterol Res Pract 2017: 1526981, 2017.

3. Yan Y, Fu G, Ye Y and Ming L: Exosomes participate in the carcinogenesis and the malignant behavior of gastric cancer Scand J Gastroenterol 52: 499-504, 2017.

4. Wang J, Liu Y, Sun W, Zhang Q, Gu T and Li G: Plasma exosomes as novel biomarker for the early diagnosis of gastric cancer. Cancer Biomark 21: 805-812, 2018.

5. Hou J, Jiang, Zhu L, Zhong S, Zhang H, Li J, Zhou S, Yang S, He Y, Wang D, et al: Circular RNAs and exosomes in cancer: A mysterious connection. Clin Transl Oncol 20: 1109-1116, 2018.

6. Kharaziha P, Ceder S, Li Q and Panaretakis T: Tumor cell-derived exosomes: A message in a bottle. Biochim Biophys Acta 1826 103-111, 2012

7. Gao Y, Wang J, Zheng Y, Zhang J, Chen S and Zhao F: Comprehensive identification of internal structure and alternative splicing events in circular RNAs. Nat Commun 7: 12060, 2016.

8. Wang Y and Wang Z: Efficient back splicing produces translatable circular mRNAs. RNA 21: 172-179, 2015.

9. Xia L, Song M, Sun M, Wang F and Yang C: Circular RNAs as biomarkers for cancer. Adv Exp Med Biol 1087: 171-187, 2018.

10. Sui W, Shi Z, Xue W, Ou M, Zhu Y, Chen J, Liu H, Liu F and Dai L: Circular RNA and gene expression profiles in gastric cancer based on microarray chip technology. Oncol Rep 37: 1804-1814, 2017.

11. Zhang H, Zhu L, Bai M, Liu Y, Zhan Y, Deng T, Yang H, Sun W, Wang X, Zhu K, et al: Exosomal circRNA derived from gastric tumor promotes white adipose browning by targeting the miR-133/PRDM16 pathway. Int J Cancer 144: 2501-2515, 2019.

12. Kumar D, Gupta D, Shankar S and Srivastava RK: Biomolecular characterization of exosomes released from cancer stem cells: Possible implications for biomarker and treatment of cancer. Oncotarget 6: 3280-3291, 2015.

13. Martin M: Cutadapt removes adapter sequences from high-throughput sequencing reads. EMBnet J 17: 2011.

14. Langmead B and Salzberg SL: Fast gapped-read alignment with bowtie 2. Nat Methods 9: 357-359, 2012.

15. Glažar P, Papavasileiou P and Rajewsky N: circBase: A database for circular RNAs. RNA 20: 1666-1670, 2014.

16. Huang da W, Sherman BT and Lempicki RA: Systematic and integrative analysis of large gene lists using DAVID bioinformatics resources. Nat Protoc 4: 44-57, 2009.

17. Ashburner M, Ball CA, Blake JA, Botstein D, Butler $\mathrm{H}$, Cherry JM, Davis AP, Dolinski K, Dwight SS, Eppig JT, et al Gene ontology: Tool for the unification of biology. The gene ontology consortium. Nat Genet 25: 25-29, 2000.

18. Kanehisa M and Goto S: KEGG: Kyoto encyclopedia of genes and genomes. Nucleic Acids Res 28: 27-30, 2000.

19. Livak KJ and Schmittgen TD: Analysis of relative gene expression data using real-time quantitative PCR and the 2(-Delta Delta C(T)) method. Methods 25: 402-408, 2001.

20. John B, Enright AJ, Aravin A, Tuschl T, Sander C and Marks DS Human MicroRNA targets. PLoS Biol 2: e363, 2004.

21. Shi Y, Yang F, Wei S and Xu G: Identification of key genes affecting results of hyperthermia in osteosarcoma based on integrative ChIP-Seq/TargetScan analysis. Med Sci Monit 23: 2042-2048, 2017.

22. Shannon P, Markiel A, Ozier O, Baliga NS, Wang JT, Ramage D, Amin N, Schwikowski B and Ideker T: Cytoscape: A software environment for integrated models of biomolecular interaction networks. Genome Res 13: 2498-2504, 2003.
23. Hansen TB, Jensen TI, Clausen BH, Bramsen JB, Finsen B, Damgaard CK and Kjems J: Natural RNA circles function as efficient microRNA sponges. Nature 495: 384-388, 2013.

24. Vishnoi A and Rani S: MiRNA biogenesis and regulation of diseases: An overview. Methods Mol Biol 1509: 1-10, 2017

25. Boukouris S and Mathivanan S: Exosomes in bodily fluids are a highly stable resource of disease biomarkers. Proteomics Clin Appl 9: 358-367, 2015

26. Nam S, Kim JH and Lee DH: RHOA in gastric cancer: Functional roles and therapeutic potential. Front Genet 10: 438, 2019.

27. Liu N, Zhang G, Bi F, Pan Y, Xue Y, Shi Y, Yao L, Zhao L, Zheng $\mathrm{Y}$ and Fan D: RhoC is essential for the metastasis of gastric cancer. J Mol Med (Berl) 85: 1149-1156, 2007.

28. Pan Y, Bi F, Liu N, Xue Y, Yao X, Zheng Y and Fan D: Expression of seven main Rho family members in gastric carcinoma. Biochem Biophys Res Commun 315: 686-691, 2004.

29. Hu M, Zhu S, Xiong S, Xue X and Zhou X: MicroRNAs and the PTEN/PI3K/Akt pathway in gastric cancer (Review). Oncol Rep 41: 1439-1454, 2019.

30. Klempner SJ, Myers AP and Cantley LC: What a tangled web we weave: Emerging resistance mechanisms to inhibition of the phosphoinositide 3-kinase pathway. Cancer Discov 3: 1345-1354, 2013.

31. Tran P, Nguyen C and Klempner SJ: Targeting the phosphatidylinositol-3-kinase pathway in gastric cancer: Can omics improve outcomes? Int Neurourol J 20 (Supp 2): S131-S140, 2016.

32. Yang $M$ and Huang CZ: Mitogen-activated protein kinase signaling pathway and invasion and metastasis of gastric cancer. World J Gastroenterol 21: 11673-11679, 2015.

33. Brown AR, Simmen RC and Simmen FA: The role of thyroid hormone signaling in the prevention of digestive system cancers. Int J Mol Sci 14: 16240-16257, 2013.

34. Kandemir EG, Yonem A and Narin Y: Gastric carcinoma and thyroid status. J Int Med Res 33: 222-227, 2005.

35. Aldinucci D and Casagrande N: Inhibition of the CCL5/CCR5 axis against the progression of gastric cancer. Int J Mol Sci 19: 1477,2018

36. Xue LJ, Mao XB, Ren LL and Chu XY: Inhibition of CXCL12/CXCR4 axis as a potential targeted therapy of advanced gastric carcinoma. Cancer Med 6: 1424-1436, 2017.

37. Ooi CH, Ivanova T, Wu J, Lee M, Tan IB, Tao J, Ward L, Koo JH, Gopalakrishnan V, Zhu Y, et al: Oncogenic pathway combinations predict clinical prognosis in gastric cancer. PLoS Genet 5: e1000676, 2009.

38. Korourian A, Roudi R, Shariftabrizi A, Kalantari E, Sotoodeh K and Madjd Z: Differential role of wnt signaling and base excision repair pathways in gastric adenocarcinoma aggressiveness. Clin Exp Med 17: 505-517, 2017.

39. Yousefi B, Mohammadlou M, Abdollahi M, Salek Farrokhi A, Karbalaei M, Keikha M, Kokhaei P, Valizadeh S, Rezaiemanesh A, Arabkari V and Eslami M: Epigenetic changes in gastric cancer induction by Helicobacter pylori. J Cell Physiol 234: 21770-21784, 2019.

(i) $\Theta$ This work is licensed under a Creative Commons Attribution-NonCommercial-NoDerivatives 4.0 International (CC BY-NC-ND 4.0) License. 